\title{
ALTERAÇÕES NA ESTABILIDADE DE AGREGADOS DE LATOSSOLO E ARGISSOLO EM FUNÇÃO DO MANEJO, NA ENTRELINHA DA SERINGUEIRA (Hevea brasiliensis) ${ }^{1}$
}

\author{
Adriana Aparecida Ribon², José Frederico Centurion³ ${ }^{3}$ Maria Aparecida Pessoa da Cruz Centurion ${ }^{4}$, \\ Kathleen Lourenço Fernandes ${ }^{5}$ e Victor Talles Lourenceti Hermógenes ${ }^{5}$
}

\begin{abstract}
RESUMO - Realizou-se um experimento com o propósito de avaliar o efeito de diferentes sistemas de manejo aplicados na entrelinha da seringueira (Hevea brasiliensis), na estabilidade de agregados em água de um Latossolo Vermelho, textura argilosa do Município de Jaboticabal, SP, e um Argissolo Vermelho-Amarelo, textura areia/ média do Município de Tabapuã, SP, cujos tratamentos consistiram em: roçadeira, adubo-verde perene (Pueraria phaseoloides) e gradagem. As amostras de solo foram retiradas após sete anos de aplicação desses manejos nas camadas de $0-0,1 ; 0,1-0,2 ; 0,2-0,3$; e $0,3-, 4 \mathrm{~m}$. A estabilidade de agregados foi obtida em peneira com classes de tamanhos de 8-4; 4-2; 2-1; 1-0,5; 0,5-0,125; e <0,125 mm. A Pueraria phaseoloides propiciou diferença significativa em relação aos demais sistemas de manejo na camada superficial $(10 \mathrm{~cm})$, quanto à distribuição de agregados, apresentando maior distribuição de agregados maiores no Latossolo Vermelho. O manejo com a grade dessa mesma camada, em ambos os solos, apresentou valores superiores de agregados de tamanho pequeno. A matéria orgânica dos solos apresentou relação direta e significativa com a estabilidade dos agregados maiores e relação inversa e significativa com a estabilidade de agregados menores.
\end{abstract}

Palavras-chave: Estabilidade de agregados; Manejo; Seringueira.

\section{CHANGES IN THE AGGREGATE STABILITY OF LATOSOL AND PODZOLIC ACCORDING TO THE MANAGEMENT BETWEEN THE ROWS OF RUBBER TREES (Hevea brasiliensis)}

\begin{abstract}
A trial was carried out with the purpose of evaluating the effect of different management systems applied between the rows of rubber trees (Hevea brasiliensis) on the aggregate stability in water of Dark Red Latosol, with clayey texture from the Municipality of Jaboticabal (SP) and Yellow Red Podzolic, sandy/ medium texture fromthe Municipality of Tabapuã (SP). The treatments consisted of disk harrowing, Pueraria phaseoloides and mowing. The soil samples were withdrawn after seven years of application of these management systems from depths of 0-0.1, 0.1-0.2, 0.2-0.3, and 0.3-0.4 $\mathrm{m}$. The stability of the aggregates was obtained in sieves with size classes of 8-4, 4-2, 2-1, 1-0.5, 0.5-0.125, and $<0.125 \mathrm{~mm}$. The Pueraria phaseoloides gave a significant difference regarding the distribution of the aggregates on the superficial layer $(10 \mathrm{~cm})$ when compared to the other management systems, presenting a higher distribution of larger sized aggregates on the Dark Red Latosol. The tillage management for this same depth presented higher values of small sized aggregates on both soils. The organic matter on the soils showed a direct and significant relation to the stability of the larger sized aggregates and an inverse relation with the stability of the smaller sized aggregates.
\end{abstract}

Keywords: Aggregate stability; Handling; Rubber tree.

\footnotetext{
${ }^{1}$ Recebido em 11.10.2013 aceito para publicação em 22.09.2014.

${ }^{2}$ Universidade Estadual de Goiás, UNU, Palmeiras de Goiás, GO - Brasil. E-mail: <adriana.ribon@hotmail.com>.

${ }^{3}$ Universidade Estadual Paulista Júlio de Mesquita Filho, UNESP, Jaboticabal, SP - Brasil. E-mail: <jfcentur@fcav.unesp.br>. ${ }^{4}$ Departamento de Produção Vegetal, Universidade Estadual Paulista Júlio de Mesquita Filho, Faculdade de Ciências Agrárias e Veterinárias de Jaboticabal, Jaboticabal, SP - Brasil. E-mail: <cidinha@fcav.unesp.br>.

${ }^{5}$ Graduando, Departamento de Solo, Universidade Estadual de Goiás, Palmeiras de Goiás, GO - Brasil. E-mail:

<kathleen_agro@hotmail.com> e < victor_lourenceti@hotmail.com>.
} 


\section{INTRODUÇÃO}

Os diferentes sistemas de manejo exercem influência sobre a formação e estabilização dos agregados do solo. Desse modo, a constante movimentação de máquinas e implementos contribui para causar alterações no tamanho de agregados, que acaba por alterar outras características físicas, como porosidade e densidade. Portanto, o manejo adequado do solo para a cultura da seringueira visando manter ou alterar o mínimo possível suas propriedades físicas é de fundamental importância, pois seu bom desenvolvimento e rendimento dependem dessas condições físicas do solo favoráveis.

Vários autores constataram que o emprego de diferentes sistemas de cultivo provoca alterações na estabilidade de agregados do solo. Em avaliação do efeito de diferentes tipos de manejo (cultivo de canade-açúcar, pastagem, solo urbano, mata nativa e cultivo de soja com rotação de culturas de inverno) sobre as propriedades físicas do solo, incluindo a estabilidade de agregados do solo.

Demarchi et al. (2011), ao estudarem a agregação de diferentes classes de solos sob cinco sistemas de manejo (pastagens, mata nativa, cultivo de soja em rotação de culturas de inverno, cultivo de cana-de-açúcar e solo urbano), verificaram alterações na qualidade estrutural do solo, sendo a pastagem o sistema de manejo com melhor qualidade estrutural devido à cobertura vegetal presente durante todo o ano, que disponibiliza ao longo do ciclo maiores quantidades de matéria orgânica.

Agregados maiores e mais pesados garantem ao solo maior estruturação, maior porosidade, maior condutividade hidráulica e maior resistência à compressão, características de inúmeras vantagens para a sustentabilidade do sistema de produção agrícola (LLANILLO, 2007). Ao estudarem a agregação de um Latossolo Vermelho-Amarelo Distrófico, Wendling et al. (2012) observaram que o comportamento da estabilidade dos agregados foi decrescente em relação aos tratamentos estudados, tendo o Cerrado apresentado maiores agregados, seguido da floresta de pinus, pastagem e plantio direto. Enfatiza-se, assim, a importância da cobertura vegetal para manutenção da estabilidade dos agregados.

Já Llanillo et al. (2006) observaram que o plantio direto e o cultivo mínimo são as formas de manejo que menos agridem as condições naturais do solo, mesmo não se igualando as condições naturais de mata, Cerrado ou campos nativos. Esses autores afirmaram que esses sistemas de manejo estão no caminho para a sustentabilidade do sistema produtivo. Considerando um manejo sustentável como aquele que permite a manutenção das propriedades físicas do solo ou que as mantenha o mais próximo do estado natural em que o solo foi encontrado.

Bastos et al. (2005) explicaram que, para a formação dos agregados, é necessário que os coloides do solo se encontrem floculados e sejam estabilizados posteriormente por algum agente cimentante. A agregação do solo pode sofrer alterações temporárias ou permanentes em função das práticas de manejo (WOHLENBERG et al., 2004). O cultivo intensivo aliado a práticas convencionais de preparo do solo causam a degradação de sua estrutura. Dessa forma, o cultivo de espécies diferentes e práticas de manejo que adicionem matéria orgânica nos solos contribuem para o rearranjamento das partículas do solo, promovendo neste a melhoria da qualidade de agregação.

A matéria orgânica, como visto, é parâmetro diretamente relacionado com a estabilidade de agregados do solo. Diversos autores verificaram a relação entre a agregação do solo e a matéria orgânica expressa pelos teores de carbono orgânico do solo (CARPENEDO; MIELNICZUCK, 1990; PALADINI; MIELNICZUCK, 1991; CASTRO FILHO et al., 1998). Essa relação é observada porque a agregação se refere ao estado de ligação entre as partículas do solo resultante por agentes cimentantes como a argila e outros (FONTONELLE, 2006). A matéria orgânica é a principal responsável por manter a estabilidade dos agregados próxima às condições naturais (LLANILLO et al., 2006). De acordo com Callegari et al. (2006), em estudo sistema de plantio direto há recuperação do carbono orgânico no solo, promovendo melhoria no estado de agregação do solo.

Nesse contexto, este estudo teve por objetivo avaliar o efeito de sistemas de manejo aplicados na entrelinha da seringueira em um Latossolo Vermelho, textura argilosa e em Argissolo Vermelho-Amarelo textura areia/média sobre a estabilidade de agregados estáveis em água e a correlação da agregação com a matéria orgânica dos solos.

\section{MATERIAL E MÉTODOS}

Foram conduzidos dois experimentos, um em Latossolo Vermelho (LV), textura argilosa, no Município

Revista Árvore, Viçosa-MG, v.38, n.6, p.1065-1071, 2014

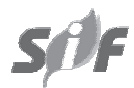


de Jaboticabal, SP (latitude: $21^{\circ} 15^{\prime} \mathrm{S}$; longitude: $48^{\circ}$ $18^{\prime} \mathrm{W}$; altitude $595 \mathrm{~m}$ ) e outro em um solo Argissolo Vermelho-Amarelo (AVA), textura arenosa/média em Tabapuã, SP (latitude: 2057’S; longitude: 49² 03’W; e altitude de $545 \mathrm{~m}$ ).

Aplicaram-se os seguintes manejos na entrelinha da seringueira (clone 235): gradagem, Pueraria phaseoloides (Kudzu tropical) e roçadeira. Esses manejos têm sido utilizados desde a implantação da cultura em 1992, com o plantio do clone PB235.

As determinações de estabilidade de agregados em água seguiram metodologia sugerida por Kemper e Chepil (1965), nas peneiras com classe de tamanho de 8-4; 4-2; 2-1; 1-0,5; 0,5-0,125; e <0,125 mm, nas profundidades de 0,0-0,1; 0,1-0,2; 0,2-0,3; e 0,3-0,4 m. Para cada camada, foram retiradas oito amostras por tratamento, por experimento, totalizando 96 amostras por experimento.

A matéria orgânica foi determinada por oxidação em solução $1 \mathrm{~N}$ de bicromato de potássio em meio ácido e titulação do excesso de bicromato em solução de sulfato ferroso amoniacal 0,5 N, usando-se difelinamina como indicador, seguindo metodologia da Embrapa (2011).
Os experimentos foram instalados em blocos ao acaso, com três tratamentos (gradagem em clone PB 235, Pueraria phaseoloides em clone PB235 e roçadeira em clone PB235), em quatro camadas de solo (0,0-0,1; $0,1-0,2 ; 0,2-0,3$; e $0,3-0,4 \mathrm{~m}$ ) e quatro repetições no Latossolo Vermelho e três tratamentos (gradagem em clone RRIM 701, Pueraria phaseoloides em clone RRIM 701 e roçadeira em clone RRIM 701), em quatro camadas de solo (0,0-0,1; 0,1-0,2; 0,2-0,3; e 0,3-0,4 m) e quatro repetições no Argissolo Vermelho-Amarelo.

A parcela experimental foi constituída por 28 plantas (quatro linhas com sete plantas), sendo as 10 plantas centrais consideradas como parcela útil.

Realizaram-se análises de variância pelo emprego do teste F e pelo teste de Tukey a 0,05 de significância, para comparação de médias dos tratamentos por peneira, nas diferentes profundidades do solo.

\section{RESULTADOS}

A distribuição de agregados no LV a AV encontrase expressa nas Tabelas 1 e 2 . O manejo com Pueraria apresentou diferença significativa em relação à roçadeira e à grade nas camadas superficiais $(20 \mathrm{~cm})$, quanto à distribuição de agregados, apresentando maior estabilidade de agregados maiores (8-4 mm).

Tabela 1 - Distribuição percentual de agregados, por classe de tamanho e profundidades analisadas, dos diferentes sistemas de manejo aplicados no Latossolo Vermelho-Escuro.

Table 1 - Percentage distribution of aggregates by size class and depths analyzed for the different management systems applied in Dark Red Latosol.

\begin{tabular}{|c|c|c|c|c|c|c|c|c|}
\hline \multirow[t]{2}{*}{ Tratamento } & \multirow{2}{*}{$\begin{array}{l}\text { Prof. } \\
(\mathrm{cm})\end{array}$} & \multicolumn{7}{|c|}{ Classe de Tamanho $(\mathrm{mm})$} \\
\hline & & 8-4 & 4-2 & $2-1$ & $1-0,5$ & $0,5-0,25$ & $0,25-0,125$ & $<0,125$ \\
\hline Roçadeira & & $39,9 b^{(1)}$ & $10,9 \mathrm{~b}$ & $7,8 \mathrm{~b}$ & $11,2 \mathrm{ab}$ & 12,0 a & 7,8 a) & 7,2 a \\
\hline Pueraria & $0-10$ & 60,4 a & $9,91 \mathrm{~b}$ & $6,2 \mathrm{~b}$ & 7,2 b & $6,4 \mathrm{~b}$ & $3,0 \mathrm{~b}$ & 5,1 a \\
\hline Grade & & $22,6 \mathrm{c}$ & 16,5 a & 12,1 a & 14,5 a & 15,6 a & 10,0 a & 7,5 a \\
\hline$\overline{C V}(\%)$ & & 47,2 & 37,7 & 46,4 & 52,8 & 52,4 & 45,3 & 39,1 \\
\hline Roçadeira & & 19,6 a & 15,4 a & 13,9 a & 16,0 a & 13,5 a & 9,9 a & $7,8 \mathrm{a}$ \\
\hline Pueraria & $10-20$ & 18,4 a & $16,4 \mathrm{a}$ & 14,5 a & 15,6 a & 15,3 a & 9,8 a & $7,4 \mathrm{a}$ \\
\hline Grade & & 18,5 a & 15,0 a & 15,5 a & 17,6 a & 15,3 a & 9,6 а & 8,8 a \\
\hline CV (\%) & & 36,9 & 27,7 & 35,8 & 24,9 & 34,1 & 46,7 & 33,5 \\
\hline Roçadeira & & 14,9 a & $16,2 \mathrm{a}$ & 15,5 a & 17,5 a & $16,4 \mathrm{ab}$ & 9,8 a & $8,2 \mathrm{a}$ \\
\hline Pueraria & $20-30$ & 13,3 a & 16,0 a & 14,9 a & 18,6 a & $15,5 \mathrm{~b}$ & 9,0 a & 9,7 a \\
\hline Grade & & 10,3 a & 15,9 a & 14,2 a & 19,0 a & 18,8 а & 10,7 a & 8,8 a \\
\hline CV (\%) & & 37,1 & 29,3 & 24,7 & 24,0 & 20,1 & 30,5 & 25,6 \\
\hline Roçadeira & & 16,5 a & 16,2 a & $12,8 \mathrm{a}$ & 15,8 a & 16,9 a & $10,6 \mathrm{a}$ & 9,8 a \\
\hline Pueraria & $30-40$ & 14,3 a & 15,6 a & 14,3 a & 18,2 a & 18,6 а & $11,1 \mathrm{a}$ & 8,7 a \\
\hline Grade & & 17,1 a & 13,2 a & $14,0 \mathrm{a}$ & 16,8 a & 19,9 а & 12,9 a & 7,1 a \\
\hline CV (\%) & & 59,9 & 36,5 & 32,7 & 20,7 & 29,2 & 41,1 & 41,1 \\
\hline
\end{tabular}

(1) Médias seguidas por uma mesma letra, na coluna, dentro das profundidades amostradas, não diferem entre si, pelo teste de Tukey a 5\%.

(1) Means followed by the same letter in the column within the soil depths, do not differ at 5\% Tukey test.

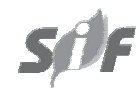

Revista Árvore, Viçosa-MG, v.38, n.6, p.1065-1071, 2014 
O manejo com grade da acamada de $10-20 \mathrm{~cm}$ propiciou valores superiores estatisticamente significativos de agregados de tamanho pequeno, tanto no LV quanto no AV.

Nas camadas mais inferiores (20-40 m), que receberam a grade e a roçadeira, os agregados apresentaram-se mais estáveis na classe 8-4 mm, em relação à Pueraria (Figura 1).

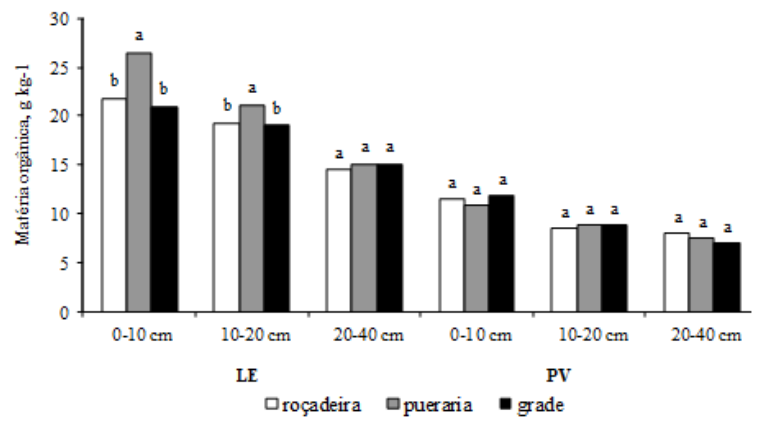

Figura 1 - Valores médios de matéria orgânica obtidos do Latossolo Vermelho e Argissolo Vermelho-Amarelo, nos tratamentos propostos.

Figure 1 - Mean values of organic matter obtained for the Dark Red Latosol and Yellow Red Podzolic in the proposed treatments.
Não ocorreram diferenças significativas entre as médias do AV entre os três tratamentos estudados, apesar das diferenças marcantes encontradas entre as médias (Tabela 2).

Com relação à estabilidade de agregados e à matéria orgânica dos solos, a distribuição de agregados na classe de 8-4 mm apresentou correlação direta e significativa com o teor de matéria orgânica (Tabela 3).

\section{DISCUSSÃO}

No Latossolo Vermelho, a maior estabilidade de agregados maiores provavelmente ocorreu devido ao maior conteúdo de matéria orgânica do solo apresentado pelo emprego da Pueraria nas camadas de 0 -10 e 10-20 cm em relação aos outros sistemas de manejo (Figura 1). Isso mostra o efeito favorável do uso da cobertura vegetal na agregação das partículas do solo, pelo fornecimento de matéria orgânica, que atua como agente agregante.

Verificou-se que o manejo com grade proporcionou maiores agregados, indicando que a manipulação intensiva do solo contribuiu para promover modificações no tamanho dos agregados do solo, provocando aumento na proporção de poros pequenos em relação aos grandes.

Tabela 2 - Distribuição percentual de agregados, por classe de tamanho e profundidades analisadas, nos diferentes sistemas de manejo aplicados no Argissolo Vermelho-Amarelo.

Table 2 - Percentage distribution of aggregates by size class and depths analyzed for the different management systems applied in the Yellow Red Podzolic.

\begin{tabular}{|c|c|c|c|c|c|c|c|c|}
\hline \multirow[t]{2}{*}{ Tratamento } & \multirow{2}{*}{$\begin{array}{l}\text { Prof. } \\
(\mathrm{cm})\end{array}$} & \multicolumn{7}{|c|}{ Classe de Tamanho (mm) } \\
\hline & & 8-4 & 4-2 & 2-1 & $1-0,5$ & $0,5-0,25$ & $0,25-0,125$ & $<0,125$ \\
\hline Roçadeira & & $50,8 \mathrm{a}^{(1)}$ & $9,2^{(1)} \mathrm{a}$ & $2,5 \mathrm{a}^{(1)}$ & $3,1 \mathrm{a}^{(1)}$ & $6,8 \mathrm{a}^{(1)}$ & $17,2 \mathrm{a}^{(1)}$ & $8,2 \mathrm{a}^{(1)}$ \\
\hline Pueraria & $0-10$ & 53,1 a & 8,9 а & 1,7 a & 2,9 a & 8,8 a & 13,6 а & 7,1 a \\
\hline Grade & & 42,1 a & 5,9 a & 0,8 a & $2,1 \mathrm{a}$ & 13,1 a & 24,0 a & $11,7 \mathrm{a}$ \\
\hline CV (\%) & & 38,0 & 20,8 & 54,3 & 57,7 & 48,8 & 47,1 a & 58,4 \\
\hline Roçadeira & & 29,7 a & 15,4 a & 7,5 a & 10,9 a & 14,9 a & $11,2 \mathrm{~b}$ & 17,2 a \\
\hline Pueraria & $10-20$ & 23,8 a & $9,4 \mathrm{ab}$ & 5,7 a & 14,4 a & 20,1 a & $20,7 \mathrm{ab}$ & 10,1 a \\
\hline Grade & & 6,6 a & $6,3 \mathrm{~b}$ & 3,20 a & 6,4 a & 11,8 a & 30,5 a & $11,0 \mathrm{a}$ \\
\hline CV (\%) & & 59,7 & 33,0 & 40,6 & 40,6 & 42,0 & 35,3 & 45,2 \\
\hline Roçadeira & & $4,6 \mathrm{a}$ & $4,6 \mathrm{a}$ & $2,7 \mathrm{a}$ & $10,1 \mathrm{a}$ & 21,7 a & 38,9 a & 14,1 a \\
\hline Pueraria & $20-30$ & $2,5 \mathrm{a}$ & $3,5 \mathrm{~b}$ & 2,5 a & 9,9 а & 25,5 a & $42,8 \mathrm{a}$ & 11,9 a \\
\hline Grade & & 6,9 a & $3,6 \mathrm{ab}$ & 4,6 a & 9,8 а & 18,8 a & 37,5 a & 15,0 a \\
\hline CV (\%) & & 53,5 & 11,8 & 47,5 & 41,9 & 30,0 & 22,7 & 32,9 \\
\hline Roçadeira & & $4,3 \mathrm{a}$ & 4,0 a & $2,7 \mathrm{a}$ & 6,9 a & 21,6 a & 42,6 a & 17,9 a \\
\hline Pueraria & $30-40$ & 2,0 a & 1,3 a & $1,4 \mathrm{a}$ & $2,4 \mathrm{~b}$ & 21,0 a & 51,3 a & 19,2 a \\
\hline Grade & & 1,6 a & $1,5 \mathrm{a}$ & $1,1 \mathrm{a}$ & $3,3 \mathrm{ab}$ & 18,4 a & 50,9 a & 22,9 a \\
\hline CV (\%) & & 54,5 & 44,1 & 45,3 & 46,0 & 26,9 & 15,5 & 31,7 \\
\hline
\end{tabular}

(1) Médias seguidas por uma mesma letra na coluna, dentro das profundidades amostradas, não diferem entre si, pelo teste de Tukey a $5 \%$.

(1) Means followed by the same letter in the column within the soil depths, do not differ at 5\% Tukey test.

Revista Árvore, Viçosa-MG, v.38, n.6, p.1065-1071, 2014 
Tabela 3 - Coeficientes de correlação dos agregados nas diferentes classes de tamanho, com o teor de matéria orgânica na profundidade de $0-40 \mathrm{~cm}$, dos solos LE e PV, nos três sistemas de manejos empregados.

Table 3 - Correlation coefficients of the aggregates in different size classes with the organic matter content in the 0-40 $\mathrm{cm}$ depth, of the LE and PV soilsin the three management systems employed.

\begin{tabular}{|c|c|c|c|c|c|c|c|}
\hline \multirow{3}{*}{ Tratamentos } & \multicolumn{7}{|c|}{ Classes de tamanho dos agregados (mm) } \\
\hline & \multicolumn{7}{|c|}{ Latossolo Vermelho-Escuro } \\
\hline & $8-4$ & $4-2$ & $2-1$ & $1-0,5$ & $0,5-0,25$ & $0,25-0,125$ & $<0,125$ \\
\hline Roçadeira & $0,87^{* *}$ & $-0,88^{* *}$ & $-0,80^{* *}$ & $-0,53^{* *}$ & $-0,88^{* *}$ & $-0,57^{* *}$ & $-0,79^{* *}$ \\
\hline Pueraria & $0,90^{* *}$ & $-0,80^{* *}$ & $-0,88^{* *}$ & $-0,86^{* *}$ & $-0,92^{* *}$ & $-0,75^{* *}$ & $-0,93^{* *}$ \\
\hline \multirow[t]{2}{*}{ Grade } & $0,83^{* *}$ & $0,21^{\mathrm{ns}}$ & $-0,24^{\mathrm{ns}}$ & $-0,27^{\mathrm{ns}}$ & $-0,79^{* *}$ & $-0,58^{* *}$ & $-0,38^{*}$ \\
\hline & \multicolumn{7}{|c|}{ Podzólico Vermelho-Amarelo } \\
\hline Roçadeira & $0,91^{* *}$ & $0,33^{\mathrm{ns}}$ & $-0,23^{\mathrm{ns}}$ & $-0,45^{* *}$ & $-0,58^{* *}$ & $-0,47^{* *}$ & $-0,53^{* *}$ \\
\hline Pueraria & $0,99^{* *}$ & $0,80^{* *}$ & $0,13^{\text {ns }}$ & $0,00^{\mathrm{ns}}$ & $-0,27^{\mathrm{ns}}$ & $-0,70^{* *}$ & $-0,53^{* *}$ \\
\hline Grade & $0,94^{* *}$ & $0,77^{* *}$ & $-0,38^{*}$ & $-0,28^{\mathrm{ns}}$ & $-0,04^{\mathrm{ns}}$ & $-0,40^{*}$ & $-0,51^{* *}$ \\
\hline
\end{tabular}

** Significativo a $1 \%$ de probabilidade; *Significativo a $5 \%$ de probabilidade; e ns - Não significativo.

** Significativo a $1 \%$ de probabilidade; *Significativo a $5 \%$ de probabilidade; e ns - Não significativo.

Resultados semelhantes foram obtidos por Calonego e Roselom (2008) em sistema de plantio direto, que contribuiu para o aumento dos teores de carbono orgânico e, consequentemente, para o aumento da estabilidade de agregados. Já sistemas convencionais promovem a dispersão das partículas do solo e a diminuição dos índices de agregados. Demarchi et al. (2011), ao estudarem a agregação do solo, observaram que os maiores agregados foram encontrados na pastagem, maiores até que em Cerrado nativo, provavelmente pela quantidade de raízes e de resíduos orgânicos das gramíneas. Castro Filho et al. (1998) citaram que as raízes das plantas estimulam a agregação estável do solo pela promoção de alta população microbiana na rizosfera, como pelo suprimento de resíduos orgânicos; por serem fontes ativas de exsudatos orgânicos, os quais são prováveis agentes efetivos na estabilização dos agregados do solo.

Calonego e Rossolem (2008) observaram que o preparo do solo com escarificação apresentou menor percentual de agregados menores que $2 \mathrm{~mm}$ em relação aos tratamentos com rotação de culturas que apresentaram agregados maiores. Portanto, o manejo dos solos com implementos deve ser feito de forma adequada, de modo a não destruir a estrutura do solo, que pode prejudicar o desenvolvimento das culturas. Corrêa (2002) observou que em sistema de plantio que se faz uso de grade há maior fracionamento de agregados. De acordo com esse autor, o uso contínuo desse implemento associado a baixos níveis de argila do solo associado ao monocultivo promove maiores alterações na estrutura do solo.
Os agregados estáveis de maior tamanho são responsáveis por proporcionar boa estrutura ao solo, promovendo maiores espaços porosos para o desenvolvimento de raízes, da fauna e da circulação de ar e água (FERREIRA et al., 2010). Sendo essas condições alcançadas com sistemas de manejo como pastagens permanentes ou com rotação de cultura ou lavouras com rotações de culturas e, ou, o uso de sistema de plantio direto, ou seja, práticas de manejo que favorecem a formação de agregados de maior tamanho (SALTON et al., 2008).

Castro Filho et al. (1998) afirmaram que o mesmo tipo de manejo altera as características do solo de forma diferente, de acordo com a profundidade do solo, como também foi observado neste estudo.

Carpenedo e Mielniczuk (1990) atribuíram à formação de agregados maiores em Latossolo Roxo sob plantio direto às forças de compressão e não por ação biológica das raízes e microrganismos, devido à superfície compactada pelo tráfego. Portanto, pode-se atribuir a formação de agregados maiores na roçadeira em comparação com a grade pelas forças de compressão devido ao uso do implemento, dando origem às camadas compactadas na superfície. A falta de mobilização do solo no tratamento com a roçadeira manteve a estabilidade desses agregados.

Paladini e Mielniczuck (1991) constataram que a falta de significância, mesmo com grandes diferenças entre as médias da porcentagem de agregados apresentadas pelos diferentes sistemas de culturas, se deve, provavelmente, ao elevado coeficiente de 
variação da característica estudada. Desse modo, tal afirmação pode ser usada para explicar os resultados da porcentagem de agregados no solo, independentemente da classe de tamanho e das profundidades analisadas de ambos os solos, pois onde as médias apresentaram diferenças marcantes, porém não significativas, os coeficientes de variação foram elevados (Tabelas 1 e 2).

Quanto à relação entre a matéria orgânica e a estabilidade dos agregados, os resultados destes corroboraram aqueles apresentados por Bastos et al. (2005), Calegari et al. (2006), Salton et al. (2008) e Zalamena (2009), os quais mostraram os efeitos positivos da matéria orgânica, representada pelo teor de carbono orgânico, sobre a agregação do solo.

O melhor coeficiente de correlação foi apresentado pela Pueraria, tanto no Latossolo Vermelho quanto no Argissolo Vermelho-Amarelo em razão, provavelmente, do maior teor de matéria orgânica do solo apresentado por esse tratamento.

A distribuição de agregados nas classes menores que $0,5 \mathrm{~mm}$, tanto no LV quanto no AV, apresentou correlação inversa com o teor de matéria orgânica, mostrando que o efeito favorável da matéria orgânica se dá sobre os agregados de maior tamanho.

Wohlenberg et al. (2004), avaliando a estabilidade dos agregados sob a influência dos sistemas de culturas em Argissolo Vermelho-Amarelo, observaram relação direta das culturas na formação e estabilização de agregados, em que a estabilidade e distribuição do tamanho dos agregados maiores em sistemas de cultivo que fornecem material orgânico e cobertura do solo o ano todo. Calonego e Rosolem (2008) verificaram que a escarificação do solo e a ausência do cultivo de plantas de cobertura proporcionaram menores porcentagens de agregados maiores que $2 \mathrm{~mm}$.

Castro Filho et al. (1998) encontraram resultados semelhantes ao notarem o efeito favorável da matéria orgânica em agregados maiores. Esses autores verificaram correlação negativa do carbono orgânico com as classes de agregados $<0,25 \mathrm{~mm}$. De acordo com estes, o aumento no Corg faz que a massa de agregados de menor tamanho diminuía, refletindo, em consequência, aumento nas classes de maior diâmetro e, portanto, dos índices de agregação.

\section{CONCLUSÃO}

O manejo com a Pueraria phaseoloides propiciou diferença significativa em relação à roçadeira e à grade na camada superficial $(0-10 \mathrm{~cm})$, quanto à distribuição de agregados, apresentando maior distribuição de agregados maiores no Latossolo Vermelho.

A aplicação da grade nas camadas superficiais (0-20 cm) proporcionou, no Latossolo Vermelho e no Argissolo Vermelho-Amarelo, valores superiores de agregados de tamanho pequeno, indicando que a movimentação intensiva do solo reduziu o tamanho dos agregados.

A matéria orgânica do LV e do AV favoreceu a formação dos agregados de maior tamanho em todos os sistemas de manejo empregados.

\section{REFERÊNCIAS}

BASTOS, R. S.; MENDONÇA, E. S.; ALVAREZ, V. H.; CORRÊA, M. M.; COSTA, L. M. Formação e estabilização de agregados do solo influenciados por ciclos de umedecimento e secagem após adição de compostos orgânicos com diferentes características hidrofóbicas. Revista

Brasileira de Ciência do Solo, v.29, n.1, p.21-31, 2005.

CALEGARI, A.; CASTRO FILHO, C.; TAVARES FILHO, J.; RALISCH, R.; GUIMARÃES, M. F.

Melhoria da agregação do solo através do sistema plantio direto. Semina, v.27, n.2, p.147-158, 2006.

CALONEGO, J. C.; ROSOLEM, C. A. Estabilidade de agregados do solo após manejo com rotações de culturas e escarificação. Revista

Brasileira de Ciência do Solo, v.32, n.4, p.1399-1407, 2008.

CARPENEDO, V.; MIELNICZUK, J. Estado de agregação e qualidade de agregados de Latossolos Roxos submetidos a diferentes sistemas de manejo. Revista Brasileira de Ciência do Solo, v.14, n.1, p.99-105, 1990.

CASTRO FILHO, C.; MUZILLI, O.; PODANOSCHI, A. L. Estabilidade de agregados e sua relação com o teor de carbono orgânico num Latossolo Roxo distrófico, em função de sistemas de plantio, rotações de culturas e 
métodos de preparo das amostras. Revista Brasileira de Ciência do Solo, v.22, n.3, p.527-538, 1998.

CORRÊA, J. C. Efeito de sistemas de cultivo na estabilidade de agregados de um Latossolo Vermelho-Amarelo em Querência, MT. Pesquisa Agropecuária Brasileira, v.37, n.2, p. 203209, 2002.

DEMARCHI, J. C.; PERUSI, M. C.; PIROLI, E. L. Análise da estabilidade de agregados de solos da microbacia do Ribeirão São Domingos, Santa Cruz do Rio Pardo - SP, sob diferentes tipos de uso e ocupação. Revista Brasileira de Tecnologia Aplicada nas Ciências Agrárias, v.4, n.2, p.7-29, 2011.

\section{EMPRESA BRASILEIRA DE PESQUISA} AGROPECUÁRIA - EMBRAPA. Manual de Métodos de Análise de Solo. 2 ed. Rio de Janeiro: 2011. 225p.

FERREIRA, R. R. M.; TAVARES FILHO, J.; FERREIRA, V. M. Efeitos de sistemas de manejo de pastagens nas propriedades físicas do solo. Semina, v.31, n.4, p.913-932, 2010.

FONTENELE, W. Indicadores físicos e hídricos da qualidade de um latossolo amarelo distrófico sob diferentes sistemas de manejo no cerrado do Piauí. 2006. 52f. Dissertação (Mestrado em Agronomia) - Universidade Federal do Piauí, Teresina, 2006.

KEMPER, W. D.; CHEPIL, W. S. Size distribuition of aggregates. In: BLACK, C. A. Methods of soil analyses agronomy. Madison: American Society of Agronomy, 1965. v.9. p.499-510.

LLANILLO, R. Indicadores de sustentabilidade da produção familiar mecanizada de grãos em modalidades de plantio direto no norte do Paraná, Brasil. 2007. 108f. Tese (Doutorado em Agronomia) - Universidade Estadual de Londrina, Londrina, 2007.

LLANILO, R. F.; RICHART, A.; TAVARES FILHO, J.; GUIMARÃES, M. F.; FERREIRA, R. R. M. Evolução de propriedades físicas do solo em função dos sistemas de manejo em culturas anuais. Semina, v.27, n.2, p.205-220, 2006.

PALADINI, F. L. S.; MIELNICZUCK, J.

Distribuição do tamanho de agregados de um solo Podzólico Vermelho-Escuro afetado por sistema de culturas. Revista Brasileira de Ciência do Solo, v.15, n.3, p.135-140, 1991.

SALTON, J. C. et al. Agregação e estabilidade de agregados do solo em sistemas agropecuários em Mato Grosso Do Sul. Revista Brasileira de Ciência do Solo, v.32, n.1, p.11-21, 2008.

WENDLING, B.; VINHA-FREITAS, I. C.; OLIVEIRA, R. C.; BABATA, M. M.; BORGES, E. N. Densidade, agregação e porosidade do solo em áreas de conversão do cerrado em floresta de pinus, pastagem e plantio direto. Bioscience Journal, v.28, n.1, p.256-265, 2012.

WOHLENBERG, E. V.; REICHERT, J. M.; REINERT, D. J.; BLUME, E. Dinâmica da agregação de um solo franco-arenoso em cinco sistemas de culturas em rotação e em sucessão. Revista

Brasileira de Ciência do Solo, v.28, n.5, p.891-900, 2004.

ZALAMENA, J. Impacto do uso da terra nos atributos químicos e físicos de solos do rebordo do Planalto-RS, 2008. 79 p. Dissertação (Mestrado em Ciência do Solo) - Universidade Federal de Santa Maria, Santa Maria, 2008. 
